N. HIRANO

KODAI MATH. J.

8 (1985), 277-284

\title{
ON HOMOTOPY INVARIANCE OF THE SOLVABILITY OF NONLINEAR VARIATIONAL INEQUALITIES
}

\author{
BY NORIMICHI HIRANO
}

\section{§1. Introduction}

Let $E$ be a Banach space and $2^{E^{*}}$ the space of subsets of the dual space $E^{*}$ of $E$. Let $A$ be an operator from $E$ into $2^{E^{*}}$. $A$ is said to be monotone if

$$
\left\langle y_{1}-y_{2}, x_{1}-x_{2}\right\rangle \geqq 0
$$

for $y_{i} \in A x_{i}(i=1,2)$. Let $H$ be a nonempty closed convex subset of $E$ and $p$ be an element of $E^{*}$. An element $x_{0}$ in $E$ is said to be a solution of the variational inequality with respect to $p$ if there exists $y_{0} \in A x_{0}$ such that

$$
\left\langle y_{0}-p, x-x_{0}\right\rangle \geqq 0 \quad \text { for all } x \in H .
$$

The variational inequalities of the form (1.1) has been studied by many authors with applications to convex programming and a large class of freeboundary problems. The existence of solutions for the variational inequality (1.1) was investigated by Browder [2], Rockaffeler [7], Stampacchia [8], Takahashi [9] and others.

Our purpose in this paper is to consider invariance of the solvability of the variational inequality (1.1) under a homotopy of monotone operators. Recently Browder established a degree theory for a class of monotone type operators. In [4], he defined a homotopy of maximal monotone operators and proved homotopy invariance of the degree. In this paper we concern a homotopy of monotone operators in the sense of Browder. Our method is based on the method employed in [4] and [6].

\section{$\S 2$. Perliminaries and statement of the main result.}

Let $E$ be a reflexive Banach space and $C, K$ be nonempty closed convex subsets of $E$. Then we denote by $\partial_{C} K$ the set of $z \in K$ such that $U(z) \cap(C-K)$ $\neq \phi$ for every nighborhood of $U(z)$ of $z$ and by $i_{C} K$ the set of $z \in K$ such that $U(z) \cap(C-K)=\phi$ for some neighborhood $U(z)$ of $z$. We also denote by $\operatorname{cl}(C)$ the closure of $C$. Let $T$ be a mapping from $E$ into $2^{E^{*}}$. Then we denote by $G(T)$ the graph $G(T)=\left\{(y, x) \in E^{*} \times E: y \in T x\right\}$ of $T$ and by $R(T)$ the range of $T$, i.e.,

Received July 18, 1984 
$R(T)=\left\{y \in E^{*}: y \in T x\right.$ for some $\left.x \in E\right\}$. Let $A$ be a monotone operator from $E$ into $2^{E^{*}}$. $A$ is said to be maximal monotone if its graph $G(A)$ is not properly contained in any other graph of monotone operator from $E$ into $2^{E^{*}}$. Let $f: E \rightarrow$ $R \cup\{+\infty\}$ be a proper lower semicontinuous convex function. The subdifferential $\partial f$ of $f$ is the mapping defined by

$$
\partial f(x)=\left\{x^{*} \in E^{*}: f(x) \leqq f(u)+\left\langle x^{*}, x-u\right\rangle \text {, for all } u \in E\right\} .
$$

It is well known that $\partial f$ is maximal monotone. Let $K$ be a nonempty closed convex subset of $E$. Then the indicator function $I_{K}: E \rightarrow R \cup\{+\infty\}$ is defined by

$$
I_{K}(x)=\left\{\begin{array}{lll}
0 & \text { if } & x \in K \\
+\infty & \text { if } & x \in K .
\end{array}\right.
$$

The indicator function $I_{K}$ is a proper lower semicontinuous convex function and $x^{*} \in \partial I_{K} x$ if and only if

$$
x \in K \text { and }\left\langle x^{*}, x-u\right\rangle \geqq 0 \text { for all } u \in K .
$$

By using the subdifferential of the indicator function $I_{H}$, the variational inequality (1.1) can be rewritten as

$$
p \in A x_{0}+\partial I_{H} x_{0} .
$$

Let $A$ be a mapping from $H$ into $2^{E^{*}}$, where $E^{*}$ is endowed with its weak topology. $A$ is said to be upper semicontinuous from $H$ into $2^{E^{*}}$, if for each $x \in H$ and each neighborhood $V$ of $A x$, there exists a nighborbood $U$ of $x$ such that $A u \subset V$ for all $u \in U \cap H$. Suppose that $A$ maps bounded sets of $H$ into bounded sets of $E^{*}$ and $A x$ is closed convex subset of $E^{*}$ for each $x \in H$. Then $A$ is upper semicontiuous if and only if the graph $G(A)$ of $A$ is a closed subset of $E^{*} \times H$ (cf. [3]). Let $\{A(t): t \in[0,1]\}$ be a family of monotone operators from $H$ into $2^{E^{*}}$. Then $\{A(t): t \in[0,1]\}$ is said to be a pseudo-monotone homotopy of monotone operators from $H$ into $2^{E^{*}}$ if $\{A(t): t \in[0,1]\}$ satisfies the following condition (cf. Browder [4]):

$\left(^{*}\right)$ Let $\left\{t_{i}\right\} \subset[0,1]$ be a sequence converging to $t$ and $\left\{\left(z_{\imath}, x_{\imath}\right)\right\} \subset E^{*} \times H$ be a sequence such that $z_{i} \in A\left(t_{\imath}\right) x_{\imath}$ for each $i \geqq 1, x_{i} \rightarrow x$ weakly in $E$, and $z_{i} \rightarrow z$ weakly in $E^{*}$. Suppose that

$$
\varlimsup_{\imath \rightarrow \infty}\left\langle z_{\imath}, x_{\imath}\right\rangle \leqq\langle z, x\rangle .
$$

Then $z \in A(t) x$ and $\left\langle z_{\imath}, x_{\imath}\right\rangle \rightarrow\langle z, x\rangle$.

Remark. To add to (*), Browder [4] assumed that $0 \in A(t) 0$ and $A(t)$ is maximal monotone for each $t \in[0,1]$.

From the definition of the homotopy $\{A(t): t \in[0,1]\}$ of monotone operators, we see that for each $t \in[0,1], A(t)$ is upper-semitinuous from $H$ into $2^{E^{*}}$. Let 
$\{A(t): t \in[0,1]\}$ be a pseudo-monotone homotopy of monotone operators from $H$ into $E^{*}$. Then the pseudo-monotone homotopy $\{A(t): t \in[0,1]\}$ is said to be bounded if for each bounded subset $G$ of $H$, the set $\cup\{A(t)(G): t \in[0,1]\}$ is bounded.

The duality mapping $J$ of $E$ into $2^{E^{*}}$ is given by

$$
J(x)=\left\{x^{*} \in E^{*}:\left\langle x^{*}, x\right\rangle=\left|x^{*}\right||x|=|x|^{2}\right\}
$$

for each $x \in E$. It is well known that $J$ is a maximal monotone operator from $E$ into $2^{E^{*}}$. It is also known that every reflexive Banach space $E$ can be renormed so that $E$ and $E^{*}$ are both locally uniformly convex (cf. Diestel [5]). Let $J$ be the duality mapping corresponding to the locally uniformly convex norm of $E$. Then $J$ has the following property (see proposition 8 of [4]);

(2.4) if $x_{i} \rightarrow x$ weakly in $E$ and $\lim \left\langle J\left(x_{i}\right), x_{i}-x\right\rangle \leqq 0$, then $x_{\imath} \rightarrow x$ strongly in $E$ and $J\left(x_{\imath}\right) \rightarrow J(x)$ weakly in $E^{*}$.

We now state our main result.

THEOREM 1. Let $H$ be a closed convex subset of a reflexive Banach space $E$ and $C$ be a bounded closed convex subset of $H$ with $\imath_{H} C \neq \phi$. Let $\{A(t): t \in[0,1]\}$ be a bounded pseudo-monotone homotopy of monotone operators from $H$ into $2^{E^{*}}$ and $p \in E^{*}$. Suppose that $p \in\left(A(0)+\partial I_{H}\right)\left(i_{H} C\right)$ and $p \notin c l\left(\cup\left\{\left(A(t)+\partial I_{I I}\right)\left(\partial_{I I} C\right): t \in\right.\right.$ $[0,1]\})$. Then $p \in\left(A(t)+\partial I_{H}\right)(C)$, for all $t \in[0,1]$.

As a direct consequence of Theorem 1 , we have the following result which is due to Browder [4] in case when $0 \in A(t) 0$ for all $t \in[0,1]$.

COROLLARY. Let $G$ be a bounded convex and open subset of a reflexive Banach space $E$ and $\{A(t): t \in[0,1]\}$ be a bounded pseudo-monotone homotopy of maximal monotone operators from $E$ into $2^{E^{*}}$. Let $p \in E^{*}$. Suppose that $p \in A(0)(G)$ and $p \notin c l(\cup\{A(t)(\partial G): t \in[0,1]\}$. Then $p \in A(t)(G)$ for all $t \in[0,1]$.

\section{§ 3. Proofs.}

In this section, we first state a necessary and sufficient condition for the variational inequality (1.1) to have a solution in $H$.

THEOREM 2(cf. [6]). Let $H$ be a closed convex subset of a reflexive Banach space $E$ and $A$ be a monotone and upper-semicontınuous mapping from $H$ into $2^{E^{*}}$, where $E^{*}$ is endowed with its weak topology. Then the following conditions are equivalent.

(i) There exist $x_{0} \in H$ and $y_{0} \in A x_{0}$ such that

$$
\left\langle y_{0}, x-x_{0}\right\rangle \geqq 0, \quad \text { for all } x \in H \text {; }
$$


(ii) there exists a bounded closed convex subset $K$ of $H$ such that for each $z \in$ $\partial_{H} K$ and $w \in A z$, there exists $x \in i_{H} K$ which satisfies that $\langle w, x-z\rangle \leqq 0$.

Remark. Sufficient conditions for the existence of solutions of (1.1) were studied by several authors (cf. Browder [2], Stampacchia [8]). Theorem 2 is a version of Theorem 1 of [6] for multivalued monotone operators. The proof of Theorem 1 of [6] is still valid for Theorem 2. Then we omit the proof.

Throughout the rest of this section, we suppose that $E, H, C$, and $\{A(t): t \in$ $[0,1]\}$ satisfy the assumption in Theorem 1 . For each operator $A: E \rightarrow 2^{E^{*}}$ and each $\lambda>0$, we denote by $A_{\lambda}$ the operator from $E$ into $2^{E^{*}}$ given by $A_{\lambda}=A+(1 / \lambda) J$, where $J$ is the duality mapping from $E$ into $E^{*}$ corresponding to a norm on $E$ in which $E$ and $E^{*}$ are locally uniformly convex. In the followings, we suppose that $A: H \rightarrow 2^{E^{*}}$ is a monotone operator satisfying the following condition:

$(*)^{\prime}$ Let $\left\{\left(z_{\imath}, x_{\imath}\right)\right\} \subset G(A)$ be a sequence such that $x_{i} \rightarrow x$ weakly in $E, z_{i} \rightarrow z$ weakly in $E^{*}$ and

$$
\varlimsup_{\imath \rightarrow \infty}\left\langle z_{\imath}, x_{\imath}\right\rangle \leqq\langle z, x\rangle .
$$

Then $z \in A x$ and $\left\langle x_{\imath}, z_{\imath}\right\rangle \rightarrow\langle x, z\rangle$.

Remark. The condition $\left({ }^{*}\right)^{\prime}$ is the case when $A(t)=A$ in $\left(^{*}\right)$. It is obvious from the condition $\left(^{*}\right)$ that each $A(t)$ satisfies the condition $(*)^{\prime}$.

LEMMA 1. Let $\lambda>0$. Let $\left\{t_{i}\right\} \subset[0,1]$ be a sequence converging to $t_{0}$ and $\left\{\left(y_{\imath}, x_{2}\right)\right\} \subset E^{*} \times E$ be a sequence such that $y_{i} \in A\left(t_{\imath}\right)_{\lambda} x_{\imath}$ for each $i \geqq 1, x_{i} \rightarrow x$ weakly in $E$ and $y_{i} \rightarrow y$ weakly in $E^{*}$. Suppose further that

$$
\varlimsup_{\imath \rightarrow \infty}\left\langle y_{\imath}, x_{\imath}\right\rangle \leqq\langle y, x\rangle .
$$

Then $x_{i} \rightarrow x$ strongly in $E,\left\langle y_{\imath}, x_{\imath}\right\rangle \rightarrow\langle y, x\rangle$ and $y \in A_{\lambda} x$.

Proof. Let $\left\{z_{i}\right\} \subset E^{*}$ be a sequence such that $z_{i} \in A\left(t_{\imath}\right) x_{\imath}$ and $y_{\imath}=z_{\imath}+(1 / \lambda) J x_{\imath}$ for each $i \geqq 1$. Since $\left\{z_{i}\right\}$ is bounded, we may suppose that $z_{\imath} \rightarrow z$ weakly in $E^{*}$. Then from the assumption, we have that

$$
\varlimsup_{\imath \rightarrow \infty}\left\langle z_{i}+(1 / \lambda) J x_{\imath}, x_{\imath}\right\rangle=\varlimsup_{\lim }\left\langle y_{\imath}, x_{\imath}\right\rangle \leqq\langle y, x\rangle=\langle z, x\rangle+\varlimsup_{\imath \rightarrow \infty}\left\langle(1 / \lambda) J x_{\imath}, x\right\rangle .
$$

Then since $\overline{\lim }\left\langle J x_{\imath}, x_{\imath}\right\rangle=\left.\varlimsup|\overline{\lim }| x_{\imath}\right|^{2} \geqq|x|^{2}$, the inequality (3.1) implies that $\overline{\lim }\left\langle z_{\imath}, x_{\imath}\right\rangle$ $\leqq\langle z, x\rangle$. Then from the condition $\left.{ }^{*}\right)$ we have that $z \in A\left(t_{0}\right) x$ and $\left\langle z_{\imath}, x_{\imath}\right\rangle \rightarrow\langle z, x\rangle$. Then again by (3.1), it follows that $\left|x_{i}\right|^{2} \rightarrow|x|^{2}$, or equivalently $\varlimsup \lim \left\langle J x_{2}, x_{i}-x\right\rangle$ $\leqq 0$. Then by (2.4), we have that $x_{i} \rightarrow x$ strongly in $E$. This completes the proof.

LEMma 2. Let $\lambda>0$ and $p \in E^{*}$. Suppose that $p \notin c l\left(\left(A_{\lambda}+\partial I_{H}\right)\left(\partial_{H} C\right)\right)$. Then the following conditions are equivalent;

(a) $p \in\left(A_{\lambda}+\partial I_{H}\right)\left(i_{H} C\right)$; 
(b) there exists $\delta>0$ such that

$$
\inf _{y \in C}\langle z-p, y-x\rangle<-\delta, \quad \text { for all } x \in \partial_{H} C \text { and } z \in A_{\lambda} x .
$$

Proof. Suppose that (b) holds. Then it is easy to see that for each $x \in \partial_{H} C$ and $z \in A_{\lambda} x$, there exists $y \in i_{H} C$ such that $\langle z-p, y-x\rangle \leqq 0$. Then by Theorem 2 , we have that (a) holds. We next suppese that (a) holds. For the sake of simplicity of the proof, we assume that $p=0, \quad 0 \in i_{H} C$ and $0 \in A_{\lambda} 0+\partial I_{H} 0$. We first show that

$$
\inf _{y \in C}\langle z, y-x\rangle<0 \quad \text { for all } x \in \partial_{H} C \text { and } z \in A_{\lambda} x .
$$

Suppose that (3.3) is false, i.e., there exist $x_{0} \in \partial_{H} C$ and $z_{0} \in A_{\lambda} x_{0}$ such that

$$
\left\langle z_{0}, y-x_{0}\right\rangle \geqq 0 \quad \text { for all } y \in C .
$$

Then since $0 \in C$, we have that $\left\langle z_{0}, x_{0}\right\rangle \leqq 0$. Suppose that $\left\langle z_{0}, x_{0}\right\rangle=0$. Let $y$ be an arbitrary element of $H$. Then since $0 \in i_{H} C$, there exists $t>0$ such that $t y \in C$. Then we have that

$$
\left\langle z_{0}, y\right\rangle=(1 / t)\left\langle z_{0}, t y\right\rangle \geqq(1 / t)\left\langle z_{0}, x_{0}\right\rangle=\left\langle z_{0}, x_{0}\right\rangle=0 .
$$

Hence from the observation above, we obtain that $\left\langle z_{0}, y-x_{0}\right\rangle \geqq 0$ for all $y \in H$, i. e., $0 \in\left(A_{\lambda}+\partial I_{H}\right)\left(\partial_{H} C\right)$. This contradicts the assumption. Therefore we find that $\left\langle z_{0}, x_{0}\right\rangle<0$. On the other hand, we have, from the assumption, that there exists $w \in A_{\lambda} 0$ such that $\langle w, y\rangle \geqq 0$ for all $y \in C$. Then from the monotonicity of $A_{\lambda}$ and the observation above, we find

$$
0 \leqq\left\langle z_{0}-w, x_{0}\right\rangle=\left\langle z_{0}, x_{0}\right\rangle-\left\langle w, x_{0}\right\rangle<0 .
$$

This is a contradiction. Thus we obtain that (3.3) holds. We now show that (3.2) holds for some $\delta>0$. Suppose that (3.2) does not hold for any $\delta>0$. Then there exist sequences $\left\{x_{i}\right\} \subset \partial_{H} C$ and $\left\{z_{i}\right\} \subset E^{*}$ such that $z_{i} \in A_{\lambda} x_{2}$ for each $i \geqq 1$ and

$$
\lim _{\imath \rightarrow \infty} \inf _{y \in C}\left\langle z_{\imath}, y-x_{\imath}\right\rangle \geqq 0 .
$$

We may suppose that $x_{i} \rightarrow x_{0}$ weakly in $E$ and $z_{i} \rightarrow z_{0}$ weakly in $E^{*}$. By putting $y=x_{0}$ in (3.4), we have that $\overline{\lim }\left\langle z_{\imath}, x_{0}-x_{\imath}\right\rangle \geqq 0$. Then by applying Lemma 1 in case when $A(t)=A$ for $t \in[0,1]$, we have that $x_{i} \rightarrow x_{0}$ strongly in $E$ and $z_{0} \in A_{\lambda} x_{0}$. Then (3.4) implies that $\inf _{y \in C}\left\langle z_{0}, y-x_{0}\right\rangle \geqq 0$. While we have by (3.3) that $\inf _{y \in C}\left\langle z_{0}\right.$, $y-x_{0}><0$. This is a contradiction. Thus we have shown that (3.2) holds.

LEMMA 3. Let $p \in E^{*}$ and $n_{0} \geqq 1$. Let $\left\{p_{n} \in E^{*}: n \geqq n_{0}\right\}$ be a sequence such that $\lim _{n \rightarrow \infty} p_{n}=p$ and $p_{n} \in\left(A_{n}+\partial I_{H}\right)(C)$ for each $n \geqq n_{0}$. Then $p \in\left(A+\partial I_{H}\right)(C)$. 
Proof. From the assumption, we have that for each $n \geqq n_{0}$, there exists $\left(z_{n}, x_{n}\right) \subset G(A)$ such that

$$
\left\langle z_{n}+\frac{1}{n} J x_{n}-p_{n}, y-x_{n}\right\rangle \geqq 0 \quad \text { for all } y \in H .
$$

Since $\left\{x_{n}\right\}$ and $\left\{z_{n}\right\}$ are bounded, we may assume that $x_{n} \rightarrow x_{0}$ weakly in $E$ and $z_{n} \rightarrow z_{0}$ weakly in $E^{*}$. Then since $p_{n} \rightarrow p$ strongly in $E$ and $(1 / n) J x_{n} \rightarrow 0$ strongly in $E^{*}$, (3.5) implies that $\overline{\lim }\left\langle z_{n}, x_{n}\right\rangle \leqq\left\langle z_{0}, x_{0}\right\rangle$. Then from the definition of $A$, it follows that $\left\langle z_{n}, x_{n}\right\rangle \rightarrow\left\langle z_{0}, x_{0}\right\rangle$ and $z_{0} \in A x_{0}$. Then again by (3.5), we have that

$$
\left\langle z_{0}-p, y-x_{0}\right\rangle \geqq 0 \quad \text { for all } y \in H,
$$

i. e., $p \in\left(A+\partial I_{H}\right)(C)$. This completes the proof.

Proof of Theorem 1. By Lemma 3, it is sufficient to show that there exists a positive integer $n_{0}$ and a sequence $\left\{p_{n}\right\}_{n \geqq n_{0}} \subset E^{*}$ such that $\lim _{n \rightarrow \infty} p_{n}=p$ and for each $n \geqq n_{0}, p_{n} \in\left(A(t)_{n}+\partial I_{H}\right)(C)$ for all $t \in[0,1]$. From the assumption, we have that there exists $r>0$ such that $B(p, r) \cap\left(A(t)+\partial I_{H}\right)\left(\partial_{H} C\right)=\phi$, for all $t \in[0,1]$, where $B(p, r) \subset E^{*}$ denotes the open ball about $p$ with radius $r$. Hence we choose $n_{0} \geqq 1$ such that $\left(1 / n_{0}\right) J\left(\partial_{H} C\right) \subset B(0, r / 2)$. Then we have that $B(p, r / 2) \cap\left(A(t)_{n}+\right.$ $\left.\partial I_{H}\right)\left(\partial_{H} C\right)=\phi$, for all $n \geqq n_{0}$ and $t \in[0,1]$. Let $x_{0}$ be an element of $C$ such that $p \in\left(A(0)+\partial I_{H}\right) x_{0}$. We put $p_{n}=p+(1 / n) J\left(x_{0}\right)$, for each $n \geqq n_{0}$. Then $p_{n} \in\left(A(0)_{n}+\right.$ $\left.\partial I_{H}\right)(C)$ for each $n \geqq n_{0}$. Also we have that for each $n \geqq n_{0}, p_{n} \notin\left(\left(A(t)_{n}+\partial I_{H}\right)\left(\partial_{H} C\right)\right)$ for all $t \in[0,1]$. We now fix $n \geqq n_{0}$ and show that $p_{n} \in\left(A(t)_{n}+\partial I_{H}\right)(C)$ for all $t \in[0,1]$. Put $t_{0}=\sup \left\{t \in[0,1]: p_{n} \in\left(A(t)_{n}+\partial I_{H}\right)(C)\right\}$. Then there exist a sequence $\left\{t_{i}\right\} \subset\left[0, t_{0}\right]$ and a sequence $\left\{\left(x_{\imath}, z_{\imath}\right)\right\} \subset E \times E^{*}$ such that $\lim _{i \rightarrow \infty} t_{i}=t_{0}, z_{i} \in$ $A\left(t_{\imath}\right)_{n} x_{\imath}$ for each $i \geqq 1$ and

$$
p_{n} \in A\left(t_{\imath}\right)_{n} x_{i}+\partial I_{H} x_{\imath} \quad \text { for all } i \geqq 1 .
$$

The equation (3.6) can be rewritten as

$$
\text { for each } i \geqq 0, \quad\left\langle z_{i}-p_{n}, y-x_{\imath}\right\rangle \geqq 0 \text { for all } y \in H .
$$

Since $\left\{x_{i}\right\}$ and $\left\{z_{i}\right\}$ are bounded, we may assume without any loss of generality that $x_{i} \rightarrow x_{0}$ weakly in $E$ and $z_{i} \rightarrow z_{0}$ weakly in $E^{*}$. Then from the definition of pseudo-monotone homotopy, we find that $z_{0} \in A\left(t_{0}\right)_{n} x_{0}$ and $\left\langle z_{2}, x_{2}\right\rangle \rightarrow\left\langle z_{0}, x_{0}\right\rangle$. Then again by (3.7), we obtain that $\left\langle z_{0}-p_{n}, y-x_{0}\right\rangle \geqq 0$ for all $y \in H$, i. e., $p_{n} \in A\left(t_{0}\right)_{n} x_{0}$ $+\partial I_{H} x_{0}$. Thus we have that $p_{n} \in\left(A\left(t_{0}\right)_{n}+\partial I_{H}\right)(C)$. Hence we claim that $t_{0}=1$. Suppose that $t_{0}<1$. Since $p_{n} \in\left(A\left(t_{0}\right)_{n}+\partial I_{H}\right)(C)$, we have by Lemma 2 that there exists $\delta>0$ such that

$$
\inf _{y \in C}\left\langle z-p_{n}, y-x\right\rangle<-\delta
$$

for all $x \in \partial_{I I} C$ and $z \in A\left(t_{0}\right)_{n} x$. Then we show that there exists $t \in\left(t_{0}, 1\right]$ such that for some $\delta^{\prime}>0$, 


$$
\inf _{y \in C}\left\langle z-p_{n}, y-x\right\rangle<-\delta^{\prime}, \quad \text { for } \quad x \in \partial_{H} C \text { and } z \in A(t)_{n} x .
$$

Suppose that (3.9) does not hold for any $\delta^{\prime}>0$. Then there exist a sequence $\left\{t_{i}\right\} \subset\left(t_{0}, 1\right]$ converging to $t_{0}$ and a sequence $\left\{\left(x_{i}, z_{2}\right)\right\} \subset H \times E^{*}$ such that $x_{i} \in \partial_{H} C$, $z_{i} \in A\left(t_{2}\right)_{n} x_{i}$ for each $i \geqq 1$ and

$$
\lim _{i \rightarrow \infty} \inf _{y \in C}\left\langle z_{i}-p_{n}, y-x_{\imath}\right\rangle \geqq 0 .
$$

Since $\left\{x_{i}\right\}$ and $\left\{z_{i}\right\}$ are bounded, we may assume that $x_{i} \rightarrow x_{0}$ weakly in $E$ and $z_{i} \rightarrow z_{0}$ weakly in $E^{*}$. Then by putting $y=x_{0}$ in (3.10), it follows that $z_{0} \in A\left(t_{0}\right)_{n} x_{0}$, $\left\langle z_{\imath}, x_{\imath}\right\rangle \rightarrow\left\langle z_{0}, x_{0}\right\rangle$ and $x_{i} \rightarrow x_{0}$ strongly in $E$. Then $x_{0} \in \partial_{H} C$. Also we have by (3.10) that

$$
\inf _{y \in C}\left\langle z_{0}-p_{n}, y-x_{0}\right\rangle \geqq 0 .
$$

Since $x_{0} \in \partial_{H} C$, this contradicts (3.8). Thus we obtain that there exists $t \in\left(t_{0}, 1\right]$ such that (3.9) holds for some $\delta^{\prime}>0$. Then by Lemma 2, we have that $p_{n} \in$ $\left(A(t)_{n}+\partial I_{H}\right)(C)$. This contradicts the definition of $t_{0}$. Thus we have shown that $t_{0}=1$, i. e., $p_{n} \in\left(A(1)_{n}+\partial I_{H}\right)(C)$.

Let $s \in(0,1)$. We put $A^{s}(t)=A(s t)$ for each $t \in[0,1]$. Then $\left\{A^{s}(t): t \in[0,1]\right\}$ is also a pseudo-monotone homotopy of monotone operators. It is easy to see that our argument above is still valid for $\{A(t): t \in[0,1]\}$ replaced by $\left\{A^{s}(t)\right.$ : $t \in[0,1]\}$. Thus we have that $p_{n} \in\left(A^{s}(1)_{n}+\partial I_{H}\right)(C)$ for each $s \in(0,1)$. This implies that $p_{n} \in\left(A(t)_{n}+\partial I_{H}\right)(C)$ for all $t \in[0,1]$. This completes the proof.

Acknowledgement. The author wishes his heartly thanks to Professor W. Takahashi for his suggestions and advice in the course of preparing this paper.

\section{REFERENCES}

[1] V. Barbu and Th. Precupanu, Convexity and optimization in Banach spaces, Editura Academiei R.S. R., Bucharest, 1975.

[2] F.E. Browder, Nonlinear variational inequalities and maximal monotone mappings in Banach spaces, Math. Ann., 183 (1969), 213-231.

[3] F.E. BRowDER, Nonlinear Operators and Nonlinear Equations of Evolution in Banacn spaces, Proceedings of Symposia in Pure Mathematics, XVIII (2), 1976.

[4] F.E. BRowDER, Fixed point theory and nonlinear problems, Bull. Amer. Math. Soc., 9 (1983), 1-41.

[5] J. Diestel, Geometry of Banach spaces, Lecture note in Mathematics, Springer Verlag 485 (1975).

[6] N. Hirano and W. Takahashi, Existence theorems on unbounded sets in Banach spaces, Proc. Amer. Math. Soc., 80 (1980), 647-650.

[7] R.T. Rockafellar, Convex functions, monotone operators and variational in equalities, Theory and Applications of Monotone Operators, Oderisi, Gubbio, 1969, 35-65.

[8] G. Stampacchia, Variational inequalities, Theory and Applications of Monotone 
Operators, Oderisi, Gubbio, 1969, 20-34.

[9] W. TAKAHASHI, Nonlinear variational inequalities and fixed point theorems, J. Math. Soc. Japan, 28 (1976), 168-181.

Department of Mathematics

FACULTY OF ENGINEERING

Yokohama National University

156, Tokiwadai, Hodogaya

YOKOHAMA, JAPAN 